\title{
The impact of the nursing practice environment on missed nursing care
}

\author{
Amanda J. Hessels ${ }^{* 1}$, Linda Flynn ${ }^{2}$, Jeannie P. Cimiotti ${ }^{3}$, Edna Cadmus ${ }^{4}$, Robyn R.M. Gershon ${ }^{5}$ \\ ${ }^{1}$ School of Nursing, Columbia University, New York, USA \\ ${ }^{2}$ College of Nursing, University of Colorado Denver, Colorado, USA \\ ${ }^{3}$ College of Nursing, University of Florida, Florida, USA \\ ${ }^{4}$ School of Nursing, Rutgers University, New Jersey, USA \\ ${ }^{5}$ Department of Epidemiology and Biostatistics, University of California, San Francisco California, USA
}

Received: May 22, 2015

DOI: $10.5430 /$ cns.v3n $4 p 60$
Accepted: August 2, $2015 \quad$ Online Published: August 16, 2015

URL: http://dx.doi.org/10.5430/cns.v3n4p60

\begin{abstract}
Objective: Missed nursing care is an emerging problem negatively impacting patient outcomes. There are gaps in our knowledge of factors associated with missed nursing care. The aim of this study was to determine the relationship between the nursing practice environment and missed nursing care in acute care hospitals.

Methods: This is a secondary analysis of cross sectional data from a survey of over 7,000 nurses from 70 hospitals on workplace and process of care. Ordinary least squares and multiple regression models were constructed to examine the relationship between the nursing practice environment and missed nursing care while controlling for characteristics of nurses and hospitals.

Results: Nurses missed delivering a significant amount of necessary patient care (10\%-27\%). Inadequate staffing and inadequate resources were the practice environment factors most strongly associated with missed nursing care events.

Conclusions: This multi-site study examined the risk and risk factors associated with missed nursing care. Improvements targeting modifiable risk factors may reduce the risk of missed nursing care.
\end{abstract}

Key Words: Missed nursing care, Practice environment, Hospital

\section{INTRODUCTION}

With over 2.7 million RN's and over 700,000 licensed practical and vocation nurses in the U.S, nurses represent the largest sector of the healthcare workforce. ${ }^{[1,2]}$ The effective delivery of front line nursing care impacts both patient safety and patient satisfaction with care. ${ }^{[3-6]}$ A new concept, referred to as "missed nursing care", defined as necessary nursing care that is omitted, either in part or whole, or delayed ${ }^{[7]}$ A number of studies have demonstrated that missed nursing care events are prevalent in US and European hospitals. ${ }^{[7-9]}$ In the US, it is estimated $28 \%$ of all necessary nursing care is believed to go undone during any given shift, and the concept of missed nursing care is emerging as an important indicator of both patient safety and patient satisfaction. $^{[7,8,10,11]}$ For instance, certain adverse outcomes have been found to be associated with missed nursing care, including higher occurrence of infections and falls, new onset delirium, pneumonia, increased length of stay, delayed discharge, increased pain and discomfort, and malnourishment. ${ }^{[8-10,12]}$ Missed nursing care events span a wide range of basic hospital nursing responsibilities, such as patient assessment (44\%), therapeutic interventions and basic care

\footnotetext{
*Correspondence: Amanda J. Hessels; Email: ah3269@cumc.columbia.edu; Address: School of Nursing, Columbia University, 630 West 168th Street, New York, NY, USA 10032, USA.
} 
$(73 \%)$, and discharge planning $(71 \%) .{ }^{[12]}$ However, information on the risk factors for missed nursing care is lacking, thus limiting our ability to develop and implement targeted interventions to reduce the risk of missed nursing care.

\subsection{Theoretical framework}

The Missed Nursing Care Model is a theoretical framework designed to predict relationships among the practice environment, nursing processes, and patient outcomes (see Figure 1). ${ }^{[13,14]}$ Missed nursing care is conceptualized as a process measure directly related to patient outcomes; when necessary nursing care is not completed, quality of care is compromised. ${ }^{[9,12]}$ Examples of necessary nursing care include patient ambulation, patient hygiene, patient teaching, patient feeding and surveillance for changes in condition. ${ }^{[12,14]}$ The model specifically posits that features of the organizational work environment impact nursing processes of care and decision making with respect to which nursing tasks are left undone, in turn leading to negative patient outcomes. When there are time constraints, individual nurses must prioritize the aspects of care rendered or omitted. ${ }^{[7]}$ These decisions are made in the context of the nursing practice environment, defined as "organizational characteristics of a work setting that facilitate or constrain professional nursing practice."[7,15] That is, hospital work settings have both organizational and professional practice characteristics. ${ }^{[16,17]}$ These include material resource allocation (including availability of medications, supplies and functioning equipment); labor resource allocation (including number and type of nurses, nurse competencies, education level); and inter-professional relationships, communication, and teamwork. ${ }^{[18]}$ Organizational support for professional nursing practice specifically includes modifiable core traits, defined as: (1) supportive front line manager; (2) adequate resources; (3) foundations for quality care; (4) nurse participation in organizational decision making and (5) collaborative relationships with physicians. ${ }^{[15,16]}$ Organizational support can influence nursing priority decision making, and therefore is hypothesized to impact what the nurse does or does not do in terms of patient care.

\begin{tabular}{|c|c|c|}
\hline $\begin{array}{l}\text { Hospital characteristics: } \\
\text { Nursing Practice environment } \\
\text { Bed size } \\
\text { Teaching status } \\
\text { Hospital teaching and } \\
\text { technology } \\
\text { Hospital geographic category } \\
\text { Staff characteristics: } \\
\text { Nurse staffing } \\
\text { Nurse education }\end{array}$ & $\begin{array}{l}\text { Missed Nursing Care: } \\
\text { Adequate surveillance (direct } \\
\text { observation/monitoring) of patients } \\
\text { Teaching of patients or family } \\
\text { Preparing patients and families for discharge } \\
\text { comforting/talking with patients } \\
\text { Adequately document nursing care } \\
\text { Administering medications on time } \\
\text { Skin care } \\
\text { Oral hygiene } \\
\text { Pain management } \\
\text { Treatment and procedures } \\
\text { Coordinating care } \\
\text { Developing or updating nursing care plans }\end{array}$ & $\begin{array}{l}\text { Patient } \\
\text { outcomes }\end{array}$ \\
\hline
\end{tabular}

Figure 1. Conceptual model guiding this study adapted from: Missed nursing care model in Kalisch BJ, et al. ${ }^{[13]}$

\subsection{Study purpose}

Despite the strong theoretical rationale, the growing interest in the concept of missed nursing care and an increasing number of studies demonstrating a significant relationship between missed nursing care and patient outcomes or between the nursing practice environment and patient outcomes, significant gaps remain. Understanding the relationship between the nursing practice environment and missed nursing care may provide insight on modifiable risk factors, thereby leading to better patient outcomes. If missed nursing care serves as mediator between features of the nursing practice environment and patient outcomes, understanding the characteristics and impact of missed nursing care will be informative. A specific knowledge gap is the identification of the specific nursing practice environment factors that are associated with missed nursing care. A secondary gap is the robust exploration of this relationship using psychometrically sound instrumentation in a large hospital sample. To address these gaps in our knowledge, we sought to explore the relationship between specific factors of the nursing practice environment and missed nursing care. We hypothesized that a supportive practice environment would be positively associated with a reduced risk of missed nursing care in acute care hospitals.

\section{MeTHODS}

This study was approved by the Rutgers University Institutional Review Board prior to study implementation. 


\subsection{Design and sample}

Data from a 2006 anonymous cross-sectional survey of 7,000 acute care hospital nurses from one US state (New Jersey) were analyzed. $50 \%$ of the nurses in the state were randomly surveyed, and 50\% completed and returned the survey. All study participants provided inpatient care at the bedside. Data available for this study included detailed information on nurse demographics, workplace characteristics, and the quality of care.

\subsection{Instruments}

The nursing practice environment was measured using the Practice Environment Scale of the Nursing Work Index (PESNWI), a 5 domain, 31-item, 4-point Likert-type (ranging from strongly disagree to strongly agree) instrument that asks nurses to characterize their work environment. Subscales and total scale score were aggregated to the hospital level. The 5 subscales from the PES-NWI used in this study included: nurse participation in hospital affairs ( 9 items, such as staff nurses serving on hospital committees), nursing foundations for quality care (10 items, such as having a preceptor program for new nurses), nurse manager ability, leadership, and support of nurses (5 items), staffing and resource adequacy (4 items), and collegial nurse-physician relations (3 items). ${ }^{[15]}$ Published internal consistency coefficients (Cronbach's alphas) for these subscales range from .71 to .84 . Intraclass correlation coefficients, reflecting the reliability of each nurse respondent's scoring of the PESNWI within his or her institution ranged from .86 to .96 , well within the range of generally-accepted values. Total subscale scale values above 2.5 indicate general agreement that the characteristics measured are present in the practice environment, whereas values below 2.5 indicate that they are absent. ${ }^{[19]}$

Missed nursing care was measured using a composite score on a measure referred to as "Tasks Left Undone", a scale developed by Lucero and colleagues and used in other high impact research. ${ }^{[8,20]}$ This is a 12-item scale that asks nurses to identify patient care activities on their last shift that they thought were necessary, but left undone. The scale construct validity has been demonstrated by other studies that found it is associated in the theoretically expected direction with RN staffing, quality of care, and frequency of adverse events in hospitals. ${ }^{[10,21]}$ The scale has an internal reliability coefficient of $0.73 .{ }^{[8]}$ The 12 nursing care items were: (1) adequate surveillance (direct observation/monitoring) of patients, (2) teaching of patients or family, (3) preparing patients and families for discharge, (4) comforting/talking with patients, (5) adequately document nursing care, (6) administering medications on time, (7) skin care, (8) oral hygiene, (9) pain management, (10) treatment and procedures, (11) coordinating care, and (12) developing or updating nursing care plans. The composite measure was calculated as the average count of the 12 nursing care activities left undone by each nurse respondent. These individual composite measures were then aggregated for each hospital, resulting in a percentage of unmet nursing care needs per hospital.

The control variables included: (1) nurse staffing levels, operationally defined as the ratio of patients to registered nurses in each hospital; (2) hospital size, operationalized as less than or equal to 100 beds, 101 to 250 beds, or greater than or equal to 250 beds; (3) teaching status, operationalized as the trainee-to-bed ratio, (number of medical residents and fellows) and categorized as minor teaching (less than 1:4 residents to trainee ratio) or major teaching (greater than 1:4 ratio); (4) high technology status, operationally defined as facilities with open-heart surgery, major organ transplant, or both; (5) hospital geographic categories, operationally defined based on United States rural-urban continuity codes (Rural-Urban Continuum Codes) of the county where the hospital is located; and (6) nurse education, operationally defined as the percentage of staff RNs with a baccalaureate degree in nursing or higher.

\subsection{Data analysis}

Data were analyzed using STATA/MP 12.1 software. Descriptive statistics were conducted to analyze the characteristics of the sample by Pearson correlations following ShapiroWilk tests of normality. Ordinary least squares (OLS) and multiple regression techniques with robust procedures using Huber-White sandwich variance estimators. Relationships between the nursing practice environment and possible confounding variables were assessed for multicollinearity prior to model testing. The level of significance at which the research hypotheses were tested was at .05 and standardized coefficients $(\beta)$ are reported.

\section{RESULTS}

\subsection{Demographics and sample characteristics}

The analytic sample consisted of 7,679 nurses in 70 New Jersey hospitals. The nurse sample is representative of the nurse population in the state; over $97 \%$ of the nurses in our sample are women, $34 \%$ are non-white and $7 \%$ are Hispanic or Latino. Nearly half earned a BSN degree (44\%) and slightly more than half held specialty certification (52\%). Nurses cared for, on average, 6 patients per shift. The majority of the 70 hospitals included in this study had 250 beds or more (52\%), were not high technology (75\%), and were either non-teaching (46\%) or minor teaching hospitals (43\%). 


\subsection{Nursing practice}

We examined the nursing practice environments and the type and amount of care nurses reported missing. The mean scale score, as shown in Table 1, for (PES-NWI) was 2.69 (possible range 1-4), indicating an above average combined score. The subscale with the highest score was "foundations for quality", lowest was staffing and resources. Nurses also reported missing a significant amount of care per hospital, ranging from $10 \%-27 \%$ (see Table 1).

\subsection{Nursing practice environment and missed nursing care}

The relationship between the nursing practice environment and missed nursing care was examined using OLS and multiple regression models. The nursing practice environment was found to be statistically significantly related to missed nursing care in that a better environment was related to less missed nursing care. The PES-NWI composite score and each of the five dimensions of the practice environment were found to be significant, inverse predictors of missed care ( $\beta$ estimates range -0.47 to $-0.77, p<.01$ ). This moderate to strong effect on the amount of missed nursing care was demonstrated by the following: (1) composite PES-NWI score explained $44 \%$ of the variance $(\beta=-.666, p<.01)$; (2) nursing foundations for quality of care explained $33 \%$ of the variance $(\beta=-.576, p<.01)$; (3) staffing and resource adequacy explained $60 \%$ of the variance $(\beta=-.773, p<.01)$; (4) nurse participation in hospital affairs explained $22 \%$ of the variance $(\beta=-.466, p<.01)$; (5) collegial nurse physician relationships explained $31 \%$ of the variance $(\beta=-.559, p<$ .01 ); and (6) nurse manager leadership, ability, and support of nurses explained $37 \%$ of the variance $(\beta=-.608, p<.01)$.

Table 1. Descriptive statistics of main variables by hospital $(\mathrm{N}=70)$

\begin{tabular}{|c|c|c|c|}
\hline Variable & $\mathbf{M}$ & SD & Range \\
\hline Composite Nursing Practice Environment & 2.69 & 0.19 & 2.23 to 3.08 \\
\hline \multicolumn{4}{|l|}{ Subscales } \\
\hline Staffing and resource & 2.43 & 0.23 & 1.86 to 2.88 \\
\hline Foundations for quality & 2.96 & 0.18 & 2.47 to 3.32 \\
\hline Nurse-physician relations & 2.84 & 0.19 & 2.25 to 3.15 \\
\hline Hospital affairs & 2.6 & 0.26 & 1.9 to 3.17 \\
\hline Nurse manager leadership & 2.58 & 0.19 & 2.04 to 3.00 \\
\hline Composite Missed Nursing Care & 0.17 & 0.04 & 0.10 to 0.27 \\
\hline \multicolumn{4}{|l|}{ Items } \\
\hline Comfort & 0.37 & 0.09 & 0.16 to 0.61 \\
\hline Surveillance & 0.16 & 0.05 & 0.03 to 0.32 \\
\hline Skin care & 0.13 & 0.05 & 0.04 to 0.28 \\
\hline Teaching patients/families & 0.28 & 0.08 & 0.10 to 0.51 \\
\hline Giving medication & 0.10 & 0.04 & 0.00 to 0.19 \\
\hline Documentation & 0.21 & 0.06 & 0.08 to 0.39 \\
\hline Coordinating care & 0.09 & 0.04 & 0.01 to 0.19 \\
\hline Pain management & 0.03 & 0.02 & 0.00 to 0.11 \\
\hline Oral care & 0.20 & 0.07 & 0.04 to 0.38 \\
\hline Treatments & 0.04 & 0.02 & 0.00 to 0.11 \\
\hline Preparing discharge & 0.11 & 0.04 & 0.00 to 0.20 \\
\hline Care plan & 0.33 & 0.07 & 0.19 to 0.54 \\
\hline
\end{tabular}

Note. Nursing practice environment measured on a possible 1-4 scale with > 2.5 indicating better work environment. Missed nursing care is average of 12 possible tasks left undone such that higher number indicates more necessary care left undone (each item missed $=0.08$ ).

These findings indicated that increasing the hospital score of the composite PES-NWI by one SD (0.19) predicted a $(-.666 \times .04) 2.6 \%$ decrease in the hospital level percentage of missed care. That is, for every full point increase in the hospital score on the composite PES-NWI, indicating a better work environment, there was a $13.7 \%$ decrease in the percentage of necessary care that is left undone by nurses in hospitals. Moreover, relatively small increases (less than one quarter of one point) in any one of the five modifiable nursing work environment subscale scores significantly decreased 
the percentage of missed nursing care in hospitals: (1) increasing staffing and resource adequacy by one SD (0.23) predicted a $3.1 \%$ decrease; (2) increasing nurse manager leadership, ability, and support of nurses by one SD (0.19) predicted a $2.4 \%$ decrease; (3) increasing foundations for quality measure by one SD $(0.18)$ predicted a $2.3 \%$ decrease; (4) increasing collegial nurse physician relationships by one SD (0.26) predicted a $2.2 \%$ decrease; and (5) increasing nurse participation in hospital affairs by one SD (0.26) predicted a $1.9 \%$ decrease in the percentage of missed nursing care.

\section{Discussion}

In this study, nurses missed a significant amount of necessary care, ranging between $10 \%-27 \%$. Notably, despite the central role of nurses conducting surveillance, providing therapeutic nursing interventions, and coordinating care and services to keep patients safe, we found that the most frequently missed nursing care included surveillance, patient hygiene, completing care plans and patient communication and education. ${ }^{[2]}$

Importantly, the nursing work environment explained a significant amount of the variance in missed care. This finding is consistent with prior research. ${ }^{[7-10,22]}$ Uniquely, this study also estimated the impact of subscales of the PES-NWI, including the dimension of staffing and resource adequacy in separate models, thus extending our knowledge on the specific factors that were associated with missed nursing care. We found that the nursing practice environment, as measured by the composite PES-NWI and subscales, explained $22 \%$ $60 \%$ of the variance in missed nursing care. These findings indicate that the amount of missed nursing care in hospitals can be decreased by $7.3 \%$ to $13.5 \%$ by improvements to the nursing practice environment, with the greatest effect on staffing and resource adequacy measure. This suggests that targeted interventions to improve any one of the dimensions of the nursing work environment will reduce the amount of missed nursing care. Hospital units can use local data, taking into consideration both need and resource availability, to develop targeted interventions to improve the nursing practice environment, and in doing so, nurses will be less likely to miss important patient care elements.

\subsection{Nursing implications}

This innovative study addressed significant gaps in our knowledge and advances the important nursing research goal to improve patient outcomes. The key finding is that good nursing practice environments, adequate staffing, and sufficient resources for the provision of nursing care are crucial as they have a strong impact on the delivery of quality care. These findings may be used to inform key healthcare executives and policymakers in their allocation of valuable resources. For example, informed policies that support a good nursing practice environment can be enacted at both the organizational and legislative levels. Moreover, findings from this study will be relevant to hospital administrators as they attempt to construct and deploy efficient mixes of material and human resources to support the provision of safe, error free care. Our findings are particularly relevant for direct care nurses who are engaged in efforts to improve their nursing practice environment. Importantly, these findings are derived from nurses' own anonymous reports of their practice environments and quality of care rendered. Nurses at the point of care delivery are acutely aware of the care that they render and omit, and are equally well equipped to use this information to help guide the development of solutions, through shared governance and professional practice councils.

\subsection{Limitations}

Study limitations include the cross-sectional design. The use of this study design, while helpful in studying associations between variables of interest, cannot ascertain causality. This study also used self-report survey data, and socially desirable responses cannot be ruled out. Since we asked nurses to recall missed nursing care events during their last shift, recall bias should not be a concern here. There is however the issue of generalizability; since our sample only included New Jersey nurses, this raises the issue of external validity of our findings to other states. While this study used data from a large multi-site study and used psychometrically sound instruments, replicating this study in other parts of the country and using more robust study designs is warranted to confirm these results.

\section{Conclusions}

Despite these limitations, important conclusions can be drawn. First, a significant amount of nursing care needed by patients was documented. Second, as reported by front line nurses, the nursing practice environment was related to the amount of care nurses miss. Third, in addition to the composite PES-NWI measure, each domain independently and significantly related to missed care and therefore each nursing unit may be able to specifically target areas that need improvement. By identifying modifiable features of the nursing practice environment and engaging in collaborative improvements, the combined effort of direct care nurses, nursing leadership and hospital administration, significant reductions in missed nursing care can be realized. Our ultimate shared goal is to improve patient outcomes and satisfaction by delivering higher quality care. 


\section{ACKNOWLEDGeMENTS}

This project was supported by grant number R36HS021988 from the Agency for Healthcare Research and Quality. The content is solely the responsibility of the authors and does not necessarily represent the official views of the Agency for Healthcare Research and Quality. The authors declare that they have no competing interests.

\section{REFERENCES}

[1] Bureau of Labor Statistics [Internet] (2012). Occupational Employment Statistics, May 2011. [cited 2012 Feb 20]. Available from: http://www.bls.gov/oes/current/oes292061.htm

[2] Page A. Keeping Patients Safe: Transforming the Work Environment of Nurses. [Internet] c2004 [cited 2012 Jan 16]. Available from: http://www.nap.edu/catalog.php?record_id=10851\#toc

[3] Aiken LH, Cimiotti JP, Sloane DM, et al. Effects of nurse staffing and nurse education on patient deaths in hospitals with different nurse work environments. Medical Care. 2011; 49(12): 1047-53. PMID:21945978. http://dx.doi.org/10.1097/MLR.0b013e3 $182330 \mathrm{~b} 6 \mathrm{e}$

[4] Aiken LH, Sermeus W, Van den Heeds K, et al. Patient safety, satisfaction, and quality of hospital care: cross sectional surveys of nurses and patients in 12 countries in Europe and the United States. British Medical Journal. 2012; 344: e1717. http://dx.doi.org/10.11 36/bmj .e1717

[5] Kutney-Lee A, McHugh MD, Sloane DM, et al. Nursing: A key to patient satisfaction. Health Affairs. 2009; 28(4): w669-w677.

[6] Meurier CE. Understanding the nature of errors in nursing: Using a model to analyze critical incident reports of errors which had resulted in an adverse or potentially adverse event. Journal of Advanced Nursing. 2000; 32: 202-207 PMID:10886452. http: //dx.doi.org/10.1046/j.1365-2648.2000.01444.x

[7] Kalisch BJ, Landstrom GL, Hinshaw AS. Missed nursing care: a concept analysis. J Adv Nurs. 2009; 65(7): 1509-1517. http: //dx.doi.org/10.1111/j.1365-2648.2009.05027.x

[8] Lucero RJ, Lake ET, Aiken LH. Nursing care quality and adverse events in US hospitals. J Clin Nurs. 2010; 19(15): 2185-2195. PMID:20659198. http://dx.doi.org/10.1111/j.1365-2702. $2010.03250 . x$

[9] Kalisch B, Tschanen D, Lee KH. Missed nursing care, staffing and patient falls. J Nurs Care Qual. 2011; 27(1): 6-12. PMID:21738057. http://dx.doi.org/10.1097/NCQ.0b013e318225aa23

[10] Schubert M, Glass TR, Clarke SP, et al. Rationing of nursing care and its relationship to patient outcomes: the Swiss extension of the International Hospital Outcomes Study. Int J Qual Health Care. 2008; 20(4): 227-237. PMID:18436556. http://dx.doi .org/10.1093 /intqhe/mzn017

[11] Sochalski J. Is more better? The relationship between nurse staffing and the quality of nursing care in hospitals. Med Care. 2004;
42(Suppl. 2): 67-73. PMID:14734944. http://dx.doi.org/10. 1097/01.mlr.0000109127.76128.aa

[12] Kalisch B, Landstrom G, Williams R. Missed nursing care: Errors of omission. Nurs Outlook. 2009; 57(1): 3-9. PMID:19150261 http://dx.doi.org/10.1016/j.outlook. 2008.05.007

[13] Kalisch BJ, Tschannen D, Lee H, et al. Hospital Variation in Missed Nursing Care. Am J Med Qual. 2011. http://dx. doi .org/10.11 $77 / 1062860610395929$

[14] Kalisch B, Williams R. Development and psychometric testing of a tool to measure missed nursing care. J Nurs Adm. 2009; 39(5): 211219. PMID:19423986. http://dx.doi.org/10.1097/NNA . 0b0 $13 \mathrm{e} 3181 \mathrm{a} 23 \mathrm{cf} 5$

[15] Lake ET. Development of the Practice Environment Scale of the Nursing Work Index. Res Nurs Health. 2002; 25(3): 176-186. PMID:12015780. http://dx.doi.org/10.1002/nur. 10032

[16] Aiken LH. Superior outcomes for Magnet hospitals: The evidence base. In: M.L. McClure \& A.S. Hinshaw (Eds.). Magnet Hospitals Revisited: Attraction and Retention of Professional Nurses. Washington, DC: American Nurses Publishing; 2002. 20 p.

[17] Flood AB, Fennell ML. Through the lenses of organizational sociology: the role of organizational theory and research in conceptualizing and examining our health care system. J Health Soc Behav. 1995; 154 169. PMID:7560846. http://dx.doi.org/10.2307/2626962

[18] Thomas-Hawkins C, Flynn L, Clarke S. Relationships between registered nurse staffing, processes of nursing care, and nurse-reported patient outcomes in chronic hemodialysis units. Nephrol Nurs J. 2008; 35(2): 123-131. PMID:18472681.

[19] Lake ET, Friese CR. Variations in nursing practice environments Nurs Res. 2006; 55(1): 1-9. PMID:16439923. http://dx.doi.o $\mathrm{rg} / 10.1097 / 00006199-200601000-00001$

[20] Ball JE, Murrells T, Rafferty AM, et al. Care left undone' during nursing shifts: associations with workload and perceived quality of care. BMJ QualSaf. 2014; 23: 116-125. http://dx.doi .org/10. 1136/bmjqs-2012-001767

[21] Sochalski J. Quality of care, nurse staffing, and patient outcomes. Policy Polit Nurs Pract. 2011; 2(1): 9-18. http://dx.doi.org/1 $0.1177 / 152715440100200103$

[22] Al-Kandari F, Thomas D. Factors contributing to nurse task incompletion as perceived by nurses working in Kuwait general hospitals. J Clin Nurs. 2009; 18: 3430-3440. http://dx.doi .org/10.1111 /j.1365-2702.2009.02795.x 